\title{
XXVII.
}

\section{Versuche über die Transplantation des Intermediärknorpels wachsender Röhrenknochen.}

\author{
Von
}

Prof. Helferich in Greifswald.

(Mit 6 Abbildungen im Text und Tafel IV. V.)

Auf dem 23. Chirurgencongress habe ich unter Demonstration einer grossen Menge von Präparaten einige Mittheilungen uber die Transplantation des Intermediärknorpels gemacht und eine genauere Beschreibung, sowie die Abbildung der Präparate für später in Aussicht gestellt. Diese Publication hat einige Verzögerung erlitten, zunächst weil ieh die Freude hatte, dass Herr Dr. Enderlen als mein Assistent die mikroskopische Untersuchung der einschlägigen Präparate ausfuhrte -, und dies führte wegen der Nothwendigkeit, neue Präparate herzustellen, z. Thl. grössere zeitliche Intervalle abzuwarten, und wegen der Schwierigkeit solcher Untersuchungen zu einigem Zeitverlust -, dann aus äusseren Gründen, welche mir selbst die Formulirung und Niederschrift meiner Resultate nicht gestatteten.

Der interessante Gegenstand scheint aber seit meinem Vortrage auf dem Chirurgencongress von anderer Seite eine Bearbeitung nicht erfahren zu haben, wie denn tiberhaupt über Transplantation des Intermediärknorpels meines Wissens bis jetzt Versuche noch nicht angestellt sind. Und doch handelt es sich um eine in biologischer Hinsicht hochinteressante und wichtige Frage, indem durch meine Versuche der Beweis geliefert ist, dass ein anatomisch so hoch und so vielseitig entwickeltes und physiologiseh so bedeutsames Gewebe wie der Intermediärknorpel nach der Transplantation unter günstigen Verlältnissen seine normale Entwickelung mit nur geringer Einbusse beibehalten kann. 
Versuche über die Transplantation des Intermediärknorpels u. s. w. 565

Im folgenden will ich über die Art und Weise, in welcher ich meine Versuche angestellt habe, und tiber die meiner Meinung nach beweisenden Präparate genauere Mittheilungen machen. Dagegen will ich es unterlassen, hier auf zahlreiche Variationen der Versuche einzugehen, welche ich auch angestellt habe, wie z. B.

a) die Reimplantation in umgekehrter Richtung (Epiphyse des excidirten Stiuckes gegen die Diaphyse gerichtet);

b) die Transplantation in eine in der Diaphyse eines anderen Knochens hergestellte Liicke, z. B. in die Radiusdiaphyse eines anderen;

c) totale Excision des Stückchens;

d) die Reimplantation des Stückchens, nachdem es in absolutem Alkohol oder in kochendes Wasser gehalten war. Ueber diese und andere Experimente soll später berichtet werden.

Einige Vorversuche hatten mir das bestätigt, was ohnedem zu vermuthen war, dass nämlich die Transplantation des Intermediärknorpels für sich allein nicht das Richtige sei. Vielmehr führte ich meine Versuche so aus, dass die dem Intermediärknorpel anliegenden Knochenpartien mit weggenommen und wieder mit implantirt wurden. Das excidirte Stück bestand demnach regelmässig aus dem Knorpel nebst einem Stückchen vom Diaphysenende und einem Stückchen der Epiphyse; das Diaphysenstück war regelmässig etwas länger als das Epiphysenstück, so zwar, dass Knorpel plus Epiphysenstick ungefâhr die gleiche Länge darboten wie das Diaphysenstlick für sich allein. Dabei wurde das dieses Stiickehen deckende Periost sorgfältig mit excidirt und darnach ebenso mit implantirt.

Als Operationsfeld wählte ich die mir von früheren Versuchen tuber die Folgen der Excision des Intermediärknorpels woblbekannte Partie des unteren Ulnaendes junger Kaninchen. Diese Versuchsanordnung bietet Vorzüge und Nachtheile, aber ich glaube, die Vorzüge tiberwiegen.

$\mathrm{Da}$ in einer grösseren Zahl der Versuche das hier excidirte Stückchen nach seiner völligen Lösung und Herausnahme an gleicher Stelle wieder implantirt wurde, war es von grossem Vortheil, dass der so hochgradig beschädigte und in seiner Festigkeit beeinträchtigte Knochen, die Ulna, eine Art Schiene an dem benachbarten Radius hatte. Es liess sioh hierdurch nach correcter Ausfuhrung der Operation in der grossen Mehrzahl der Fälle eine reinliche Implantation des Stüekchens bewirken und eine erwünschte Lage desselben in der Folge erhalten. Gewisse Nachtheile, welche durch diese Versuchsanordnung bedingt sind, werden später ihre Erwähnung und Erläuterung finden. 


\section{Die Ausführung der Operation.}

Das Thierchen wird am besten in linker Seitenlage so in ein Handtuch fixirt, dass nur die rechte vordere Extremität frei bleibt. Dieselbe wird nun gründlich abgeseift und mit Sublimatlösung 1:1000 desinficirt. Dann nahe an die Kante des Tisches gelagert, so dass sie uber die Tischkante herausgezogen werden kann, und gerade die Ulnarseite frei zugänglich ist. Die linke Hand des Operateurs fixirt die Pfote, seine rechte Hand ist frei fur die Incision; ein Gehülfe hält das Thier selbst, und ein zweiter Gehülfe ist zur Assistenz an der Wunde mit Häkchen bereit.

Die bei jungen Thierchen weichen und kurzen Härchen wurden nun meistens einfach so gescheitelt, dass die für die Incision bestimmte Stelle frei war, und der Hautschnitt annähernd ohne Durchtrennung von Haaren gemacht werden konnte. Einige Male wurde einige Tage vor der geplanten Operation eine Entbaarung des Operationsfeldes durch Schwefelcalciumpaste bewirkt; jedoch stellte sich hiernach bei den jungen Thierchen häufig Hautentzündung ein, welche ein Verschieben der Operation erforderte und störend war.

Nach der Ausfithrung des Hautschnittes klafft die Wunde so viel, besonders wenn ein leichter Zug der die Pfote haltenden Hand des Operateurs dazu hilft, dass alles gut zu übersehen ist. Da erkennt man die helle Zone des Intermediärknorpels und die anliegenden Knochentheile, zunächst noch von einigen feinen, weissglänzenden Sehnen bedeckt. Wenn der Hautschnitt nicht rein ulnar, sondern zugleich etwas nach der Dorsalseite zu ausgeführt wurde, erschien es am zweckmässigsten.

Zunächst müssen nun die Sehnen freipräparirt und seitwärts gelagert werden. An der ulnaren Seite nach der Volarseite zu finden sich zwei feine, beisammenliegende Sehnen von dem Extensor digiti IV proprius und dem Extensor digiti V proprius, weiter volarwärts findet sich nach deren Lösung die stärkere Sehne des Extensor carpi ulnaris. An der radialen Seite liegt die starke Sehne des Extensor digitorum communis, nach oben, der Diaphyse entsprechend. in einem kräftigen Muskelbauch, abwärts in vier feine für die zweite bis 5. Zehe bestimmte Schnen übergehend. Durch feine, ritzende und dann schlitzende Züge mit einem scharfen, feinen Messerchen lassen sich diese Sehnen alle rasch und sicher ohne weitere Hülfe freimachen. Nach aufwärts benutzte ich meistens ein stumpfes Messerchen, um schlitzend die Muskelbäuche vom Knochen abzulösen. Das Periost soll dabei möglichst wenig verletzt werden. 
Dasselbe halbstumpfe Messerchen benutzte ich nun, um im Bereich des unteren Ulnaendes, soweit es exeidirt werden sollte, in den Spalt zwischen Ulna und Radius einzugehen und eine möglichst vollständige Trennung herbeizuführen.

Während dieses letzten Actes ist es nützlich, wenn ein Gehülfe mit feinem Häkchen die Sehnen zur Seite hält, unerlässlich ist dieses für die nun vorzunehmende Durchtrennung des Knochens.

Um eine bestimmte, für die einzelnen Thierchen eines Wurfes gleiche Länge von der Ulna zu excidiren, wurde ein Zirkel mit der entsprechenden Stellung seiner Spitzen fixirt $(5-7 \mathrm{~mm})$ und dann die Spitzen in die freiliegende Partie an den erw tussohten Stellen leise eingedriekt. So entstanden an Epiphyse und Diaphyse kleine, leicht blutende Marken, welche sodann für die Ausfuhrung der Knochentrennung sehr dienlich waren.

Die Trennung habe ich nun, während die Sehnen seitwärts gehalten wurden, mit der neuen Patentscheere der Firma Henckels ausgeführt, welehe dadureh ausgezeichnet ist, dass das eine Blatt derselben gleichzeitig drückt und ziehend schneidet. Dadurch gelingt es, einen glatten Schnitt, fast ohne alle Quetschung der Theile auszuftihren. Am besten geeignet fand ich die Soheere P. 4 der Henckels'schen Liste, indem ich ihre Spitze dem Markirpunkt entsprecheud an der radialen Seite der Ulna ansetzte.

War das Stückchen abgetrennt, so wurde nun die Pfote von dem Gehtilfen gehalten, dann das Stiuckchen mit einer feinen Hakenpincette gefasst, ein wenig in die Höhe gehoben und endlich die wenigen noch fixirenden Weichtheilfasern auf der Unterseite völlig durchschnitten.

Nun wurde das ganze Stìckchen frei aus der Wunde herausgenommen und dann in verschiedener Weise weiter benutzt. In einer grossen Anzahl der Versuche wurde es an der gleichen Stelle sofort wieder implantirt.

Die Vollendung der kleinen Operation bestand dann darin, dass iber das sorgfältig eingelegte Sttickchen die Sehnen ron beiden Seiten wieder heribergeschoben, die Haut dann mit einer fortlaufenden Seidennaht (unter Benutzung feiner runder Nadeln) vereinigt wurde. Desinfieirende Flüssigkeit kam mit der Wunde liberhaupt nicht in Berührung.

Zum Schlusse kam Collodium und etwas weicher Mull auf die Wunde, darüber eine schmale Binde, welche mit Stecknadeln so befestigt wurde, dass dieselben gleichzeitig als Schiene die operirte Stelle schützten. 
Die freigelassenen Zehen boten in der ersten Zeit nach der Operation etwas Schwellung, doch bald benutzte das Thierchen sein Bein in normaler Weise. Die Wundheilung erlitt in der weitaus grössten Zahl der Versuche nicht die geringste Störung.

Fig. 1.

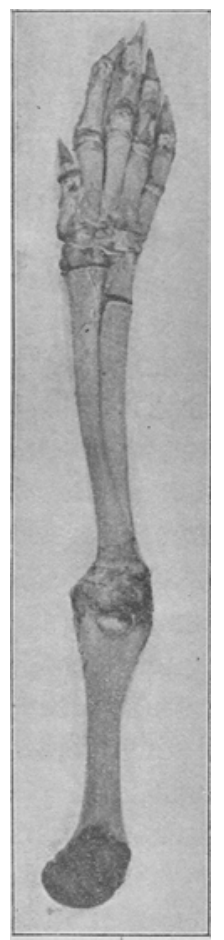

Fig. 2.

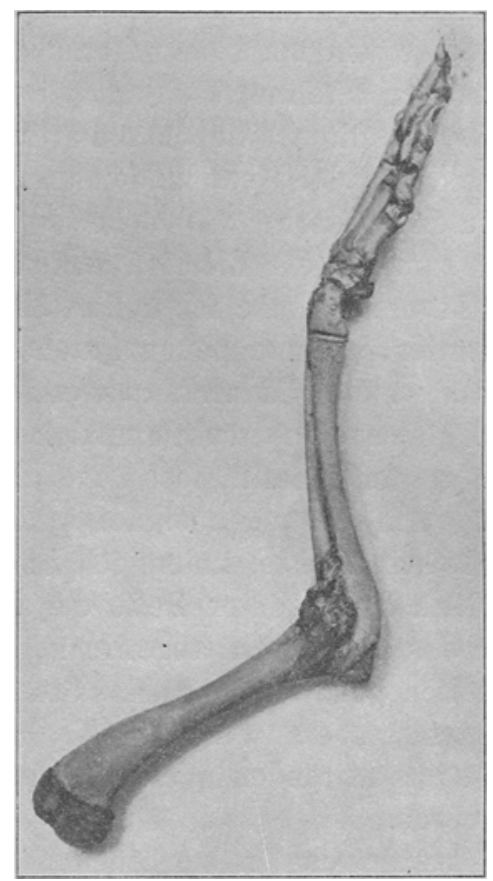

Indem nun zu der Operation die rechte vordere Extremität benutzt wurde, das Thierchen aber im übrigen intact blieb und wohl gepflegt wurde, war die normal waehsende linke vordere Extremität zum Vergleiche brauohbar. Natürlich wurden junge Kaninchen eines and desselben Wurfes immer gleichzeitig operirt, so dass auch die einzelnen 'Thierehen, wenn sie zu verschiedenen Zeiten gestorben oder getödtet waren, einen annähernden Vergleich der operirten Theile zuliessen; namentlich kann das gesammte Wachsthum der betreffenden Knochen aus der Betrachtung einer solchen Reihe gut erkannt werden.

Im ganzen habe ich an 131 Thierchen diese Transplantationsversuche ausgeführt, ungerechnet zahlreiche und mehrfach modificirte 
Versuche über die Transplantation des Intermediärknorpels u. s. w. 569

Controlversuche. Da ich sämmtliche Operationen selbst ausgefubrt habe, kann ich fur die correcte Ausfuhrung derselben einstehen.

Zunächst erseheint es mir angebracht, auf die normalen Verhältnisse der Knochen der vorderen Extremität und besonders der Ulna an der Hand einer Abbildung hinzuweisen. Die beiden Knochen des Vorderarmes liegen dicht aneinander. An ihrem peripheren Ende findet sich eine kurze Epiphyse am Radius und eine gut doppelt so lange an der Ulna. Hierdurch wird bedingt, dass die untere Epiphyse der Ulna noeh reiehlieh in ihrer halben Länge dem Diaphysenende des Radius correspondirt.

Das obere Ende der Knochen trägt nur ganz unbedeutende Epiphysen. Die obere Epiphyse der Ulna sitzt nur dem äussersten Ende des Olecranon auf, wie sich bei seitlicher Betrachtung sofort ergiebt. Das untere Humerusende artieulirt mit der Diaphyse der Ulna. Hieraus folgt, dass ein von der oberen Epiphysengrenze der Ulna ausgehendes Längenwachsthum zwar die gesammte Länge dieses Knochens vermehrt, jedoch für die Länge der ganzen Extremität nichts beiträgt. Wird das Ellbogengelenk als Mittelpunkt genommen, so ist die gesammte peripher von demselben liegende Länge des Knochens, abgesehen von der essten Anlage, ein Product des unteren Intermediärknorpels. Zudem ist es ja längst bekannt, dass die Vorderarmknochen vorwiegend oder fast ausschliesslieh an ibrem unteren (Hand-) Ende in die Länge wachsen.

Die Reimplantation des Stückchens an gleicher Stelle und in gleicher Richtung geschah immer in der oben beschriebenen Weise. Von dieser Versuchsreihe liegen 40 skeletirte, getrooknete Präparate vor, in weiteren 10 Fällen wurden die Theile zur mikroskopisehen Untersuchung verwendet.

Ich stelle folgende Reihe gelungener und, wie ich glaube, beweisender Präparate zusammen:

Prïparat 14c. Das Thierchen wurde 5 Wochen alt am 22. October 1891 mit drei anderen operirt. Das excidirte und an gleicher Stelle replantirte Stückchen hatte ein Länge von $6 \mathrm{~mm}$. Am 27. October 1891 wurde das Thierchen getödtet.

Das hier abgebildete Präparat (Fig. 3) verauschaulicht aufs beste den Zustand nach der Operation und bedarf keiner weiteren Beschreibung, doch muss daranf hingewiesen werden, dass der Defect in der Vlna dem unteren Diaphysenende des Radius entspricht, die stehengebliebene Partie der Ulnaepiphyse zeigt ziemlich genau die gleiche Ausdehnung wie die anliegende Radiusepiphyse.

Präparat 22 a stammt von einem Kaninchen, welches $41 / 2$ Wochen alt am 7. Juli 1893 operirt wurde. Die Länge des excidirten und sofort 
replantirten Stückchens betrug $7 \mathrm{~mm}$. Das Thierchen wurde am 18. Juli getödtet. Versuchsdauer also 11 Tage.

Fig. 3 (Präp. 14c).

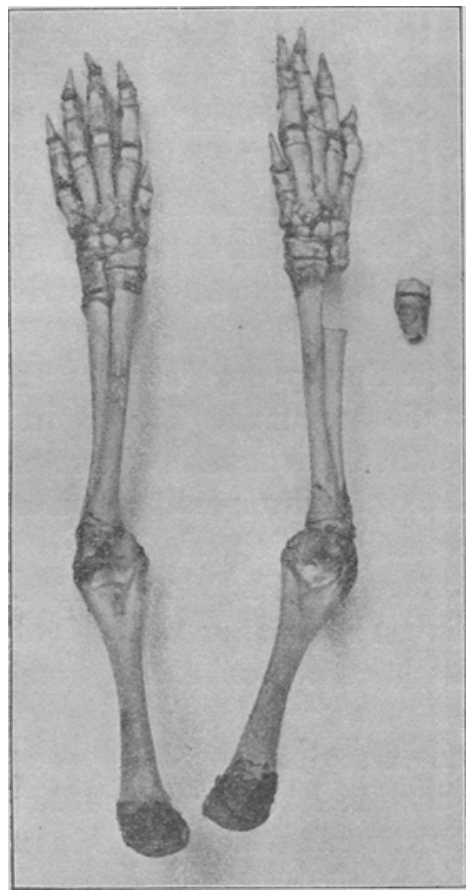

Fig. 4 (Präp. 22a).

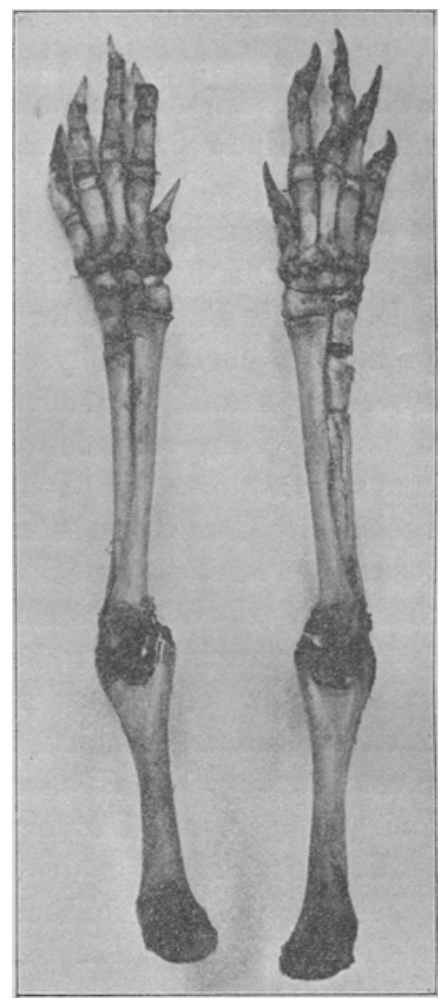

An dem Präparate (Fig. 4) zeigt sich keine Formstörung und keine messbare Längendifferenz der Vorderarmknochen. Das replantirte Stiickchen ist auffallend fest wieder verwachsen, namentlich die Epiphysenstücke sind sehr gat verheilt. Der Zusammenhang beider Stïckchen mit der Umgebung ist schon so fest, dass bei der leichten Maceration und folgenden Eintrocknung die Narben gehalten, die Fuge des Intermediärknorpels aber nachgegeben hat, dem letzteren entsprechend findet sich ein Defect, breiter als der normalen Knorpelfuge entspricht. Die Enden des replantirten Stückchens stehen jetzt $8 \mathrm{~mm}$ auseinander, eine Verlängerung, welche offenbar auf Conto einer Verdickung, resp. Verbreiterung des Intermediärknorpels zu setzen ist.

Präparat 14 d stamme von einem Thierchen des gleichen Wurfes, wie das vorher erwähnte (14c), wurde wie jenes, 5 Wochen alt, am 22. October 1891 operirt und 27. November 1891 getödtet. Versuchsdauer 36 Tage. An dem Präparat zeigt sich im Vergleich zur gesunden linken Extremität eine leichte Ulnarflexion der Pfote im Handgelenk. 
Der Radius erscheint beiderseits gleich lang, aber die rechte Ulna im ganzen und ganz besonders im Bereiche ihrer Diaphyse kürzer als die linke, dagegen die untere Ulnaepiphyse rechts etwas länger als links. Die Stelle, wo der Knochen durchtrennt war, ist an der Epiphyse nicht mehr, an der Diaphyse nur spurweise noch zu erkennen. An dem Präparat zeigt sich sofort die Verkttrzung der Diaphyse (ca. 1,5 mm), die Verlängerung der Epiphyse (ca. 0,5 mm) und die Beziehung der Ulnaepiphyse zur Radiusdiaphyse, indem die Stelle, in deren Bereich sich diese beiden Knochen berühren an dem operirten Bein $4 \mathrm{~mm}$ beträgt gegen $3 \mathrm{~mm}$ auf der gesunden linken Seite.

Im Vergleich zum Präparat $14 \mathrm{c}$ (Fig. 3) welches den Zustand gleich nach der Operation markirt und einem Thierchen desselben Wurfes von gleicher Grösse angehört, ergiebt sich, dass die operirte Ulnadiaphyse $2,75 \mathrm{~cm}$ Länge besitzt, gegen $2,4 \mathrm{~cm}$ des gesunden unverletzten Knochens zu Beginn des Versuches (bei 14c).

Präparat 21 b stammt von einem Kaninchen, welches 2 Wochen alt, am 31. Mai 1893 operirt wurde. Die Länge des excidirten und replantirten Stiickchens betrug $5 \mathrm{~mm}$ 。 Das Thierchen wurde am 19. Juli getödtet. Versuchsdauer 49 Tage.

An dem Präparate zeigt sich nur eine ganz geringe Formveränderung. Das Diaphysenstiuck ist an seinem distalen Ende etwas verdickt und an einer Stelle, welche $5^{1 / 2} \mathrm{~mm}$ oberhalb des noch deutlich sichtbaren Intermediärknorpels liegt, in einer Ausdehnung von $3 / 4 \mathrm{~cm}$ Länge mit der anliegenden Diaphyse des Radius verwachsen. Die Länge der Epiphyse an der reclten operirten Seite beträgt 7,5, an der gesunden Seite $6,5 \mathrm{~mm}$. Auch die Epiphyse ist auf der operirten Seite gegen den Intermediärknorpel zu etwas verdickt, die Gesammtlänge der Ulnadiaphyse ist rechts 3,1 , links $3,4 \mathrm{~cm}$; die Diaphyse des Radius ist rechts 3,55 , links $3,8 \mathrm{~cm}$ lang. Die Entwickelung der übrigen Knochen ist beiderseits gleich, die ganze Extremität zeigt mit Ausnahme der geringen Verkürzung an der rechten Seite keinen Unterschied.

Präparat 23 d stammt von einem Thierchen welches, 31/2 Wochen alt, am 10. Juli 1893 operirt wurde. Das excidirte und replantirte Stückchen war $6 \mathrm{~mm}$ lang. Am 5. September 1893 wurde das Thier getödtet, die Versuchsdauer ist somit 57 Tage.

An dem getrockneten Präparat ist eine grobe Formstörung nicht zu bemerken. Die Ulnadiaphyse ist an der rechten operirten Seite etwa $3 \mathrm{~mm}$ oberhalb des Intermediärknorpels in der Ausdehnung von gut $1 \mathrm{~cm}$ mit dem Diaphysenstïck des Radius verwachsen. Die Stelle des Intermediärknorpels bildet eine unebene, fast zackige Linie; an mehreren Stellen finden sich feine Knochenauflagerungen, welche zwischen Epiphyse und Diaphyse eine kleine Brücke herstellen. An der Ulna ist die Länge des Diaphysenstückes rechts 3,25, links $3,5 \mathrm{~cm}$, die Länge der Epiphyse rechts 0,9 , links $0,65 \mathrm{~cm}$; Länge der Radiusdiaphyse rechts 3,9 , links $5,05 \mathrm{~cm}$. Das Verhältniss der Ulnaepiphyse zur Radiusdiaphyse ergiebt sich aus folgenden Zahlen: an der rechten operirten Seite berührt die Ulnaepiphyse in einer Ausdehnung von 7,5 mm das andere Ende der 
Radiusdiaphyse, an der linken gesunden Seite ist dies nur in $3,5 \mathrm{~mm}$ Ausdehnung der Fall.

Zum Vergleich wird das Präparat $23 \mathrm{~b}$ herangezogen, in welchem nur eine Versuchsdauer von 23 Tagen vorliegt. Das Thierchen ist am

Fig. 5 (Präp. 23 d).

Fig. 5 (Präp. 23 b).
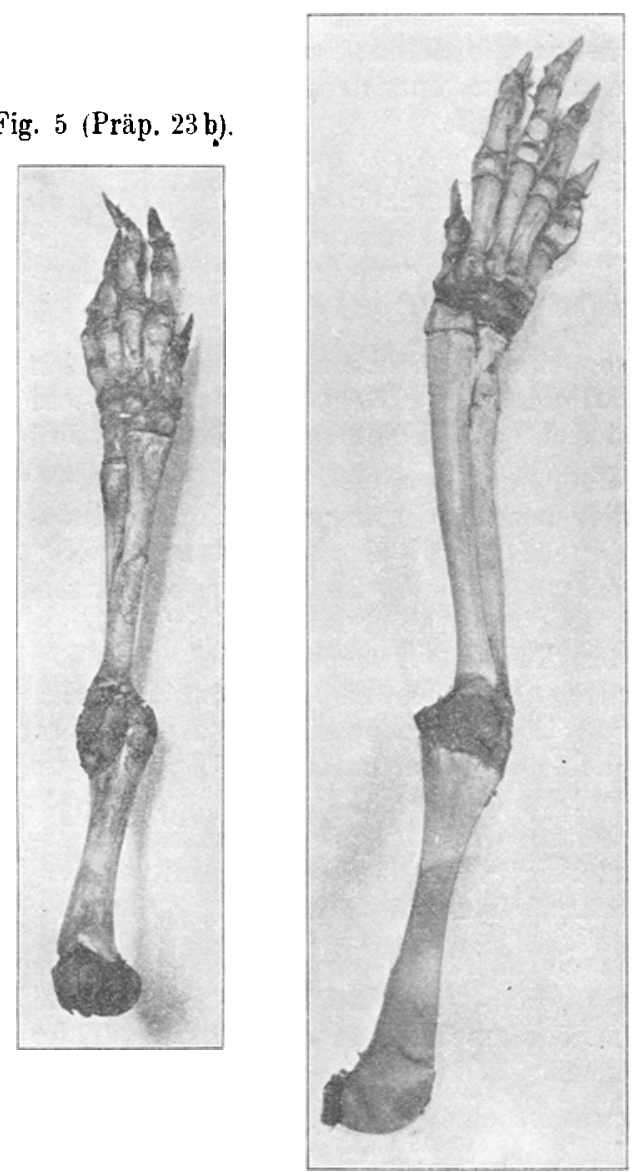
gleichen Tage operirt wie 23 d, aber schon am 2. Aug., also 23 Tage nach der Operation, getödtet worden. Vergleicht man die gesunde Extremität von $23 \mathrm{~b}$ mit der operirten von $23 d$, wie es in beistehender Abbildung möglich ist, so ergiebt sich der deutliche Unterschied und das Wachsthum an der operirten Seite.

Präparat 21 d stammt von demselben Wurf wie das an 4. Stelle beschriebene Präparat, nur ist das Thierchen erst später, nämlich am 10. October 1893, also $4^{1 / 3}$ Monat nach der Operation getödtet worden.

Dieses Präparat zeigt Verhältnisse, welche in dem Beschauer Zweifel entstehen lassen, ob hier wirklich die Excision und Replantation des Stückchens vorgenommen wurde, denn in der Form der einzelnen Knochen und in ihrem Verbältniss zu einander ist kaum ein Unterschied zu bemerken. Nur bei genauem Nachsehen ergiebt sich, dass die Ulnadiaphyse um etwa $2,5 \mathrm{~mm}$ an der rechten Seite kürzer ist als linkerseits, die rechte Ulnaepiphyse ist um ca. $2 \mathrm{~mm}$ länger als links, die Dicke und ganze Form der Knochen ist dagegen auf beiden Seiten analog. Die Berührung zwischen Ulnaepiphyse und Radiusdiaphyse ist rechts $5 \mathrm{~mm}$, links $3,5 \mathrm{~mm}$.

Bei einem Vergleich mit dem Präparat 21 b ergiebt sich das erheb. liche Wachsthum, welches die gesunde wie die operirte Extremität bei $21 \mathrm{~d}$ aufzuweisen haben. Bei der Wichtigkeit, welches gerade diesem Präparat, das den Schlass der Serie bildet, beizumessen ist, halte ich die Abbildung desselben für geboten. Auf der beigeftigten Tafel IV sind diese Extremitäten so abgebildet, dass ein Vergleich leicht möglich ist. Es stellt sich 
heraus, dass die gesunde Ulna von $3,4 \mathrm{~cm}$ bei $21 \mathrm{~b}$ auf $4,95 \mathrm{~cm}$ bei $21 \mathrm{~d}$ gewachsen ist, während die operirte Ulna sich von $3,05 \mathrm{~cm}$ bei $21 \mathrm{~b}$ bis auf $4,4 \mathrm{~cm}$ entwickelt hat.

Diese Zahlen beweisen wohl unwiderleglich, dass der Intermediärknorpel, wenn er in der oben näher angegebenen Weise herausgenommen und replantirt wird, unter günstigen Verhältnissen seine normalen Lebenseigenschaften, speciell seine Productionsfähigkeit für das Knochenwachstbum, nicht einzubüssen braucht. Eine Verminderung dieser Leistung ist sicher za constatiren, allein von einem völligen Verlust dieser Fähigkeit ist gar keine Rede.

Die Thatsache, dass die Epiphyse an der operirten Seite einen gewissen Ausgleich der eintretenden Verkürzung herbeiführt, ist nach den mitgetheilten Protokollen zweifellos; allein wer hiernach glauben oder behaupten wollte, dass dadurch allein der Ausfall des Längswachsthums ersetzt werde, würde viel zu weit gehen und den sicheren Thatsachen keine Rechnung tragen.

Von hohem Interesse sind für mich die photographischen Aufnahmen der zur mikroskopischen Untersuchung verwandten Präparate, bei deren Herstellung mich besonders Herr Dr. T en der i c h unterstuitzt hat. Man erkennt an denselben (vgl. Taf. V) in deutlicher Weise die Verdickung, resp. Schwellung des Intermediärknorpels besonders in den ersten Wochen nach der Operation. Die Knorpelreihen sind an der operirten Seite trotzdem an vielen Stellen gut erhalten und schon bei schwacher Vergrösserung erkennbar. Zum Theil sind die Knorpelreihen allerdings verwaschen und undeutlich geworden. An einem meiner Präparate (28d) ist der Intermediärknorpel ausser. ordentlich verändert und zeigt in seinem Gefüge und in seiner Umgebung Verhältnisse, wie sie an die Unordnungen bei schwerster rhachitischer Störung erinnern. Hier ist auch das weitere Wachsthum der Ulna sistirt, und der Radius entsprechend verkrïmmt.

Erklärung der Abbildungen auf Tafel IV u. V.

Tafel IV zeigt das Schlusspräparat unserer Serie $21 \mathrm{~d}$, und zwar die gesunde und die operirte Seite, daneben zum Vergleiche die gesunde Extremitåt von $21 \mathrm{~b}$ mit fast 2 Monate Lürzerer Versuchslauer.

Tafel $\nabla$ zeigt den Durchschnitt durch das untere Ende der Vorderarmknochen eines Kaninchens, welches 10 Tage nach der Operation getödtet wurde (Präparat 26g), bei schwacher Vergrösserung. Man erkennt deutlich an der operirten Seite Fig. 2a das replantirte Stückchen mit dem verbreiterten Intermediärknorpel und einer geringen Verlängerung der Epiphyse gegenüber der danebenstehenden gesunden Seite desselben Thierchens, Fig. $2 b$. 